\title{
Anti-psoriatic Agent
}

National Cancer Institute

\section{Source}

National Cancer Institute. Anti-psoriatic Agent. NCI Thesaurus. Code C29708.

Used topically or systemically, natural or synthetic Anti-psoriatic agents relieve symptoms of psoriasis by controlling rapid growth of skin cells, reducing DNA synthesis and mitotic activity in hyperplastic epidermis, and restoring normal epidermal cell proliferation and keratinization. ( $\mathrm{NCl04)}$ 